\title{
PARAMETRIC STUDY OF THE STREAK BREAKDOWN MECHANISM IN HARTMANN FLOW
}

\author{
E. Zienicke, D. Krasnov \\ Fakultat fur Maschinenbau, Technische Universitat Ilmenau, \\ PF 100565, D-98684 Ilmenau, Germany (egbert.zienicke@tu-ilmenau.de)
}

Introduction. The goal of this study is the identification of the regions of stability and instability for the laminar Hartmann flow against transition to turbulence. In previous work [1] it has been shown numerically that the transition takes place by a two step mechanism: (1) large transient growth of optimal 2D-perturbations causing a (2) secondary instability against 3D-random perturbation triggering the transition to turbulence. The mechanism leads to a streak breakdown scenario (see, e.g., [3]). With this two-step mechanism the gap of the critical Reynolds number between linear stability analysis $\left(\mathrm{R}_{\mathrm{L}}=48250\right.$, see [4]) and experimental results $\left(150<\mathrm{R}_{\mathrm{C}}<250\right.$, see references in [4]) could be closed satisfactory using direct numerical simulation. We found a critical Reynolds number of $\mathrm{R}_{\mathrm{C}}=350$ for $\mathrm{Ha}=10,20$. This is in good agreement with a recent experiment [5] giving a critical Reynolds number of $\mathrm{R}_{\mathrm{C}}=380$.

In this contribution we address two additional topics, which extend our former results to a parametric study: (1) Closure of the gap between the Hartmann number in the experiment $(130<\mathrm{Ha}<1690)$ and our numerical calculations $(\mathrm{Ha}=10,20)$. (2) Determination of the size of 2D-optimal disturbances and 3Drandom perturbations, which are necessary to trigger transition to turbulence. This gives an exact representation of the system behaviour in the region of conditional stability.

1. Numerical simulation of the streak breakdown mechanism. We consider the flow of an electrically conducting fluid between two parallel nonconducting planes inside a uniform magnetic field normal to the walls. The laminar solution is the Hartmann profile with two Hartmann layers of thickness $\delta=L / \mathrm{Ha}$ at the lower and the upper wall. Here $L$ is the channel width and $\mathrm{Ha}$ is the Hartmann number $\mathrm{Ha}=B_{0} L \sqrt{\sigma /(\rho \nu)}$. ( $B_{0}$ is the magnetic field, $\sigma$ is the electric conductivity, $\rho$ is the density, $\nu$ is the kinematic viscosity).

The non-dimensional formulation of the MHD-equations in the quasistatic approximation (for details see [1]) is based on the centreline velocity $U_{0}$, the channel width $L$ and the size of the applied magnetic field $B_{0}$ as typical scales. Considering the incompressible flow, $\nabla \cdot \mathbf{v}=0$, the following set of equations has to be solved:

$$
\begin{aligned}
\frac{\partial \mathbf{v}}{\partial t}+\mathbf{v} \cdot \nabla \mathbf{v} & =-\nabla p+\frac{1}{\operatorname{HaR}} \nabla^{2} \mathbf{v}+\frac{H a}{\mathrm{R}}\left[\left(-\nabla \phi+\mathbf{v} \times \mathbf{e}_{z}\right) \times \mathbf{e}_{z}\right], \\
\nabla^{2} \phi & =\omega_{z} .
\end{aligned}
$$

Here we have used the Reynolds number R, which is based on the thickness of the Hartmann layer. It is linked to the usual Reynolds number $\operatorname{Re}=U_{0} L / \nu$ by the relation $\mathrm{R}=\mathrm{Re} / \mathrm{Ha}$. The above governing equations are solved in a $3 \mathrm{D}$ rectangular domain with periodic boundary conditions in horizontal directions and no-slip conditions on upper and bottom walls (vertical direction). The limiting walls are electrically non-conducting that means that the normal component of the current density has to be zero. The flow is driven in the streamwise direction with a given 


\section{E. Zienicke, D. Krasnov}

flow rate. The total current in the spanwise direction integrated over the vertical direction is fixed to zero. The flow solver, which was originally developped and applied in [1], implements a pseudo-spectral approximation with a Fourier series in the horizontal directions and a Chebyshev polynomial expansion in the vertical direction.

The procedure, which realizes the two-step mechanism numerically, is implemented as follows. The two-step scenario of transition to turbulence implies that the growth of the 2D-optimal perturbations modifies the flow in such a way that it becomes unstable against perturbation by small 3D-random noise. Therefore, there are two phases of the transient process to be modeled separately, namely (1) the 2D-evolution of the optimal perturbations and (2) fully 3D-flow evolution after that. The appropriate 2D-optimal perturbations for the Hartmann flow are streamwise vortices of the size of the Hartmann boundary layer, as was computed by Gerard-Varet [6]. To trace the 2D flow evolution, the code is modified to work in a pure $2 \mathrm{D}$ mode, which is achieved by setting to zero all non-zero coefficients of the Fourier expansion in the streamwise direction in each timestep. The simulations are started with a specified initial amplitude $E_{2 D}(t=0)$ of the optimal perturbations. The 2D-flow evolves then until the energy $E_{2 D}(t)$ attains a maximum. At this moment, which is denoted here and after as $t_{\mathrm{opt}}$, the $3 \mathrm{D}$ random noise with a given amplitude $E_{3 D}$ is imposed on the modulated flow. After that the procedure of artificial suppression of non-zero Fourier coefficients is switched off and the code continues to run further with full 3D-functionality engaged.

2. Critical Reynolds number for high Hartmann number. The first issue of this work is to get numerically the link to the Hartmann numbers in the experiment of Moresco \& Alboussière [5] lying between 130 and 1690. The critical Reynolds number found in the experiment was $\mathrm{R}_{\mathrm{C}}=380$, independent on the Hartmann number, which shows that in the experiment the case of an isolated Hartmann layer is reached. Our previous runs with $\mathrm{Ha}=5,10,20$ all yielded the same critical Reynolds number of $R_{C}=350$. Mainly two questions from the comparison of experiment and numerics arose at the start of this work: (1) Does the numerical value change, if one goes to higher values of the Hartmann number? (2) When is the case of isolated Hartmann layers reached numerically? The ideal case of isolated Hartmann layers is reached for $\mathrm{Ha} \rightarrow \infty$. The higher is the Hartmann number, the thinner are the Hartmann layers and the smaller is the region of the channel they occupy. At a certain point the both Hartmann layers do not influence each other and the problem can be considered as for an isolated Hartmann layer. The critical Reynolds number then should only depend on R (see $[4])$.

In figure 1 a systematical check of stability and instability dependent on the Reynolds number $\mathrm{R}$ and the Hartmann number $\mathrm{Ha}$ is shown. The black filled triangles denote that for the corresponding $\mathrm{R}$ and $\mathrm{Ha}$ no transition to turbulence was observed with the two-step scenario, independently on how large the 2Doptimal disturbances and 3D-random perturbations were chosen. Blank triangles mark the runs, where a transition to turbulence could be found with the two-step mechanism with a certain choice of $2 \mathrm{D}$ - and $3 \mathrm{D}$-perturbations. The results shown for $\mathrm{Ha}=10,20$ were already computed for the previous publication. The critical Reynolds number is detected in the interval $345<\mathrm{R}_{\mathrm{C}}<350$. For the higher Hartmann numbers, which are considered here, the critical Reynolds number grows to $360<\mathrm{R}_{\mathrm{C}}<370$ for $\mathrm{Ha}=40$ and $380<\mathrm{R}_{\mathrm{C}}<390$ for $\mathrm{Ha}=100$. This is closer to the result of the experiment and well closes the gap between numerics and experiment. As the critical Reynolds number still varies with the Hartmann 


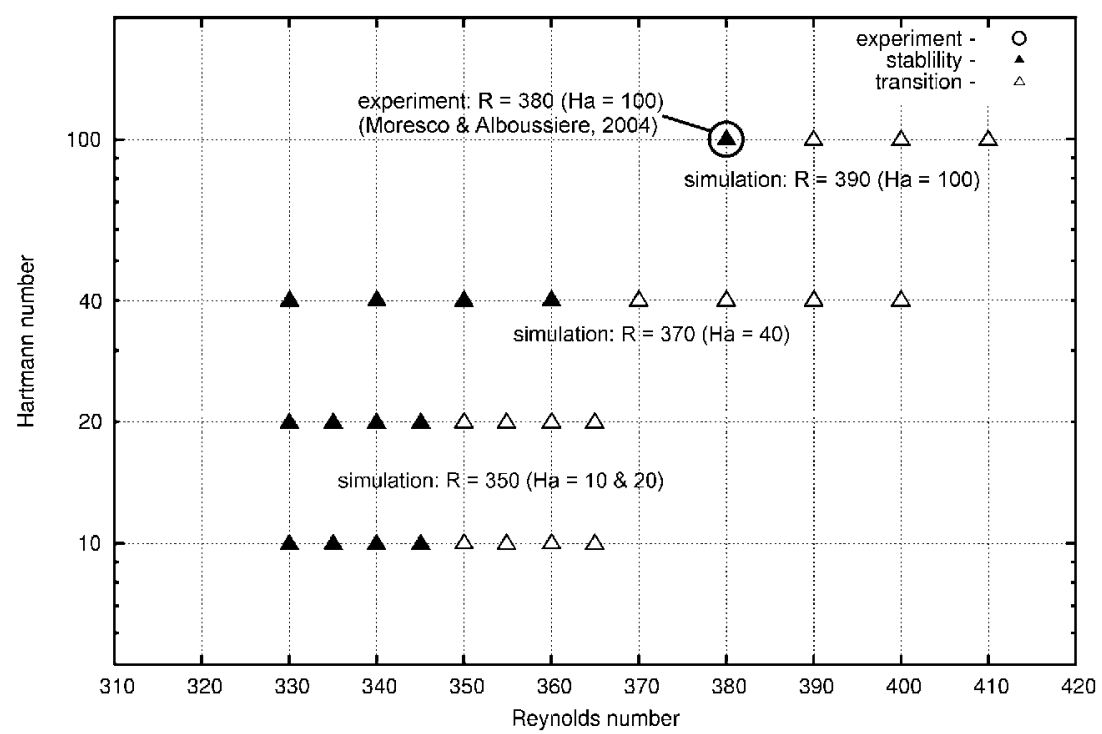

Fig. 1. Critical Reynolds number R vs Hartmann number. Filled triangles mark points (R,Ha) in the parameter space, where no transition could be detected independent on the size of $2 \mathrm{D}$ and 3D-perturbations. Empty triangles mark parameter pairs, where a transition by the streak breakdown scenario could be observed. The numerical value of the critical Reynolds number approaches the experimental value for $\mathrm{Ha}=40$ and 100 giving a better agreement with reference $[5]$.

number, the numerical calculations show that the limit of an isolated Hartmann layer is not yet reached for $\mathrm{Ha}=40$ and $\mathrm{Ha}=100$. Because one needs several runs to enclose the critical Reynolds number for a given Hartmann number, it was not possible to treat the case of higher Hartmann numbers (the next would have been $\mathrm{Ha}=200$ ) with our computational resources.

\section{Necessary size of $2 \mathrm{D}$-optimal and 3D-random perturbations.}

The second main topic of this parametric study is the determination of the necessary size of perturbations in the two-step scenario for transition to turbulence. This was lead through using the streak breakdown scenario as described in Section 2 in the Reynolds number interval $\mathrm{R} \in[350,1000]$ for $\mathrm{Ha}=10,15$ and 20 . Varying the size of $2 \mathrm{D}$-optimal disturbances and 3D-random perturbations in a wide range, we filtered out two values for each Reynolds number R: (1) The absolut minimal necessary 2D-perturbation (independent of the size of 3D-perturbations) to trigger the transition to turbulence. (2) The minimal 3D-random perturbation for the given 2D-perturbation that provides a transition to turbulence. The search for this both values was organized in two loops of runs, the inner loop varying the 3D-perturbation starting from below and the outer loop varying the size of the 2D-optimal disturbation. As it turned out, the necessary minimal perturbations change only very slightly for the three tested Hartmann number 10, 15 and 20. This allows to present the result for all three Hartmann numbers in one figure as shown in Fig. 2.

As one expects, the necessary absolute 2D-perturbations and the corresponding 3D-perturbations decrease with the increasing Reynolds number. The factor between perturbations at $\mathrm{R}=350$ and $\mathrm{R}=1000$ is $10^{-3}$ for absolute minimal 2Dperturbations and even $10^{-3.5}$ for the corresponding minimal 3D-perturbations. This means that already in this interval for the Reynolds number the necessary 


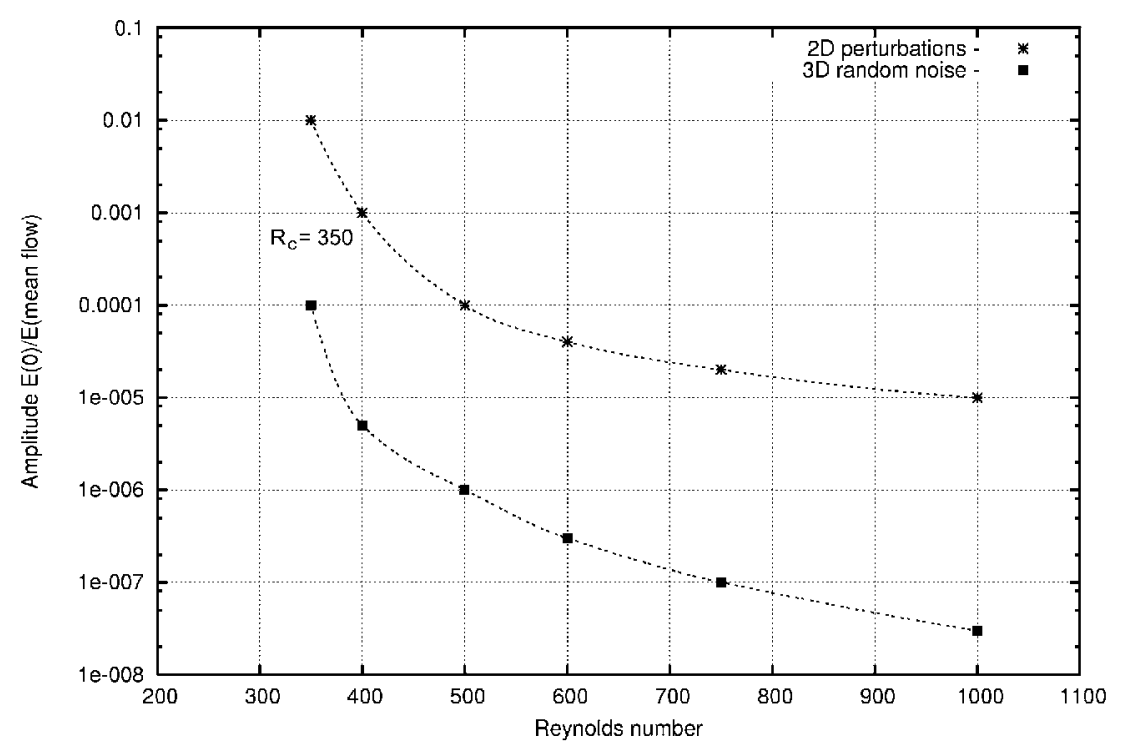

Fig. 2. Minimal sizes of 2D-optimal disturbances and 3D-random perturbations for the transition to turbulence in dependence on the Reynolds number $\mathrm{R}$ based on the Hartmann layer thickness. As the results for $\mathrm{Ha}=5,10$ and 15 are almost identical, the results for these three Hartmann numbers are summarized by the two curves in this figure.

size of perturbations is decreasing significantly.

Acknowledgments. The authors express their gratitude to the Deutsche Forschungsgemeinschaft for financial support in the frame of the "Research Group Magnetofluiddynamics" at the Ilmenau University of Technology. Access to computation time on a JUMP supercomputer was provided by the John von Neumann Institute (NIC) at the Forschungszentrum Jülich, Germany. The authors thank Thomas Boeck for the original development of the flow solver and useful comments.We are also indebted to Andre Thess and Oleg Zikanov for stimulating discussions.

\section{REFERENCES}

1. D. Krasnov, E. Zienicke, O. Zikanov, T. Boeck, A. Thess. Numerical study of the instability of the Hartmann layer. J. Fluid Mech., vol. 504 (2004), pp. 183-211.

2. D. Krasnov, E. Zienicke, O.Zikanov, T. Boeck, A. Thess. Numerical simulation of transition to turbulence in MHD-channel flow In Direct and Large-Eddy Simulation V, ed. R.Friedrich, B. Geurts and O. Metais, ERCOFTAC Series, (2004) pp. 197-204, Kluwer Academic Publishers, Dordrecht.

3. P.J.Schmid, D.S. Henningson. Stability and Transition in Shear Flows (Springer, Berlin, 2001).

4. R.J. Lingwood, T. Alboussiere. On the stability of the Hartmann layer. Phys. Fluids, vol. 11 (1999), pp. 2058-2068.

5. P. Moresco, T. Alboussière. Experimental study of the instability of the Hartmann layer. J. Fluid Mech., vol. 504 (2004), pp. 167-181.

6. D. Gerard-Varet. Amplification of small perturbations in a Hartmann layer. Phys. Fluids, vol. 14 (2002), pp. 1458-1467. 